\title{
Características zootécnicas de touros da raça Nelore submetidos a um programa de seleção para precocidade sexual
}

\author{
[Zootecnic characteristics of Nelore bulls subjected to a selection program for sexual precocity] \\ F.P.C. Lima, J.A.G. Bergmann, P.R. Xavier*, A.P. Marques $J r$ \\ Escola de Veterinária - Universidade Federal de Minas Gerais - UFMG \\ Av. Antônio Carlos, 6627 - Caixa Postal 567 \\ 30123-970 - Belo Horizonte, MG \\ RESUMO
}

\begin{abstract}
Foram utilizados 24 tourinhos da raça Nelore (Bos taurus indicus) divididos em três grupos de oito animais cada, de acordo com o perímetro escrotal (PE): Grupo 1 (G1) - PE acima de 22,8cm; Grupo 2 (G2) - PE entre 21,6 e 22,6cm; e Grupo 3 (G3) - PE entre 20,1 e 21,2cm. Para a análise da área de olho do lombo (AOL) e espessura de gordura (EG), os 24 animais foram divididos em dois grupos : G1 (mais precoces) e G2 (menos precoces), classificados de acordo com a idade em que cada animal atingiu a puberdade. $\mathrm{O}$ G1 apresentou maior $\mathrm{PE}$ à puberdade e menor peso à desmama em comparação ao $\mathrm{G} 2 \mathrm{e}$ ao G3. As medidas de AOL não apresentaram diferenças entre os grupos G1 e G2. Os animais do G1 atingiram a precocidade sexual em idade menor, enquanto a deposição de gordura maior e mais cedo ocorreu nos animais do G2, considerados mais tardios. A utilização de características zootécnicas em um programa de seleção para precocidade sexual pode constituir um avanço para a produtividade pecuária, aumentando os ganhos genéticos e diminuindo os custos de produção.
\end{abstract}

Palavras-chave: touro Nelore, precocidade sexual, seleção

\begin{abstract}
Twenty-four Nelore bulls (Bos taurus indicus) were divided in three groups of eight animals each according to EP: Group 1 -EP above average (EP>22.8cm); Group $2-E P$ 5\% below average (EP between 21.6 and 22.6cm); and Group $3-E P$ from 10 to 5\% below average (EP between 20.1 and $21.2 \mathrm{~cm}$ ). For the AOL and EG analysis the 24 animals were divided in two groups: G1 (more precocious) and G2 (less precocious), classified according to the age in which each animal reached puberty. G1 presented greater EP at puberty and less weight in comparison to G2 and G3. The AOL measures did not present difference between $G 1$ and G2 groups. The animals in G1 reached puberty at a younger age, while the fat deposition occurred in G2. The use of productive characteristics in a program to select animals for sexual precocity can constitute a great advance for cattle productivity, increasing the genetic gain and decreasing production costs.
\end{abstract}

Keywords: Nelore bull, sexual precocity, selection

\section{INTRODUÇÃO}

Os processos seletivos em pecuária de corte tropical, especialmente em bovinos de corte Bos taurus indicus, têm sido dedicados principalmente ao desenvolvimento ponderal. Entretanto, mais recentemente a atenção tem

Recebido em 11 de julho de 2011

Aceito em 29 de setembro de 2011

*Autor para correspondência (corresponding author)

E-mail: pramosxavier@yahoo.com.br se voltado também para as características reprodutivas (Ferraz e Eler, 2007).

A seleção zootécnica com base nos desempenhos reprodutivo e produtivo dos touros é relativamente recente no Brasil, especialmente entre os criadores de gado Zebu. Nos plantéis de elite, houve sempre valorização estética dos 
animais em detrimento do ganho de peso, da precocidade sexual e da qualidade de carcaça (Pereira, 2004). Esta situação está se modificando ao longo dos anos e a escolha dos indicadores de eficiência reprodutiva tem merecido mais destaque pelos pesquisadores. A reprodução é um processo complexo e a seleção direta para características ligadas a ela é difícil de ser aplicada. Alguns indicadores podem se enquadrar nesse conceito, como a seleção para perímetro escrotal, que está relacionada com a idade à puberdade em machos e fêmeas, medidas de velocidade de ganho de peso e peso à desmama e aos 12 meses de idade, que podem ser correlacionadas com puberdade e precocidade sexual (Gressler, 1998; Pereira, 2004).

Dentre as características reprodutivas, o perímetro escrotal tem sido recomendado para ser incluído nos programas de seleção de touros de corte, por estar geneticamente associado, de maneira favorável, a características seminais e ponderais, bem como a características reprodutivas das fêmeas, sugerindo que o melhoramento da fertilidade, tanto de machos quanto de fêmeas, poderá se dar por meio da seleção indireta baseada no perímetro escrotal (Fonseca et al., 1992; Bergmann et al., 1996; Quirino, 1998).

Características zootécnicas produtivas, como a área de olho de lombo (AOL), a espessura de gordura (EG) e a cobertura de gordura na garupa entre o íleo e o ísqueo (P8), podem ser utilizadas como avaliações auxiliares na busca pela determinação da precocidade sexual em touros da raça Nelore. A AOL é a medida que tem sido usada como indicativo de desenvolvimento muscular do animal, enquanto as medidas de EG e P8 são consideradas características de herdabilidades média a alta, ou seja, apresentam potencial para o melhoramento genético mediante seleção massal (Sainz et al., 2003; Ferraz e Eler, 2007).

Tuner et al. (1990), Sainz et al. (2003) e Ferraz e Eler (2007) sugerem que a EG e a P8 estariam relacionadas à precocidade de crescimento e sexual. A comprovação da existência de correlação genética favorável entre características de crescimento, reprodutivas e de carcaça, poderia auxiliar o produtor, no direcionamento para seleção de algumas características, visto que, atualmente, nos programas de melhoramento genético, existem informações genéticas de mais de quinze características de importância econômica (Bergmann et al., 1996; Pereira, 2004).

Perspectivas futuras na seleção de animais para características ligadas à reprodução devem incluir a busca por precocidade como prioritária, considerando-se o trinômio das precocidades: sexual, crescimento e acabamento. Os três tipos estão interligados, seja com correlações positivas ou negativas. $\mathrm{Na}$ adoção dos critérios para seleção, é importante escolher características com estimativa de herdabilidade alta, indicando que são influenciadas por genes de ação aditiva e que tornam a seleção um mecanismo efetivo de melhoramento. A seleção para precocidade sexual no Bos taurus indicus pode diminuir os custos de produção, reduzir o intervalo de gerações e aumentar os ganhos genéticos e a produtividade (Brito et al., 2004).

Este trabalho teve como objetivo avaliar características zootécnicas produtivas de animais submetidos a um programa de seleção para precocidade sexual e verificar possível interrelação entre elas.

\section{MATERIAL E MÉTODOS}

O trabalho foi realizado com 24 tourinhos da raça Nelore (Bos taurus indicus), pertencentes à Fazenda Rancho da Matinha, localizada em Uberaba, MG. Os animais são registrados na Associação Brasileira de Criadores de Zebu $(\mathrm{ABCZ})$ e participam do Programa de Melhoramento Genético da Raça Nelore (PMGRN). Os tourinhos foram divididos em três grupos de oito animais cada, de acordo com o perímetro escrotal $(\mathrm{PE})$ :

Grupo 1 - animais com PE acima da média (PE $>22,8 \mathrm{~cm})$

Grupo 2 - animais com PE 5\% abaixo da média até a média (PE entre 21,6 e 22,6cm); Grupo 3 animais com PE $10 \%$ até $5 \%$ abaixo da média (PE entre 20,1 e 21,2cm).

Para a análise da AOL e da EG, os 24 animais foram divididos novamente em dois grupos: o G1, constituído pelos animais mais precoces, e o $\mathrm{G} 2$, pelos menos precoces, classificados e agrupados de acordo com a idade em que cada 
animal atingiu a puberdade segundo exame andrológico (Freneau et al., 2006).

Os animais foram mantidos conforme as condições de manejo da fazenda, ou seja, os grupos foram separados em piquetes e receberam dieta à base de silagem de milho no cocho. A pesagem foi individual, a cada 30 dias, durante sete meses (12 aos 18 meses), para acompanhar o desenvolvimento ponderal dos animais. O perímetro escrotal foi mensurado uma vez a cada 30 dias, por meio de fita métrica posicionada na região de maior diâmetro do escroto, após tração de ambos os testículos na direção ventrocaudal. A espessura de gordura, a área de olho de lombo e a cobertura de gordura na garupa (EGP8) foram medidas com auxílio de ultrassom, quando os animais estavam com 15 meses de idade, por técnico treinado, utilizando-se equipamento com transdutor linear de $17,2 \mathrm{~cm}$ e $3,5 \mathrm{MHz}$, (Aloka
$500 \mathrm{~V})$ em conjunto com um sistema de captura de imagens (Blackbox, BioTronics, Ames, IA, EUA). Imagens foram coletadas, entre a $12^{\mathrm{a}}$ e $13^{\mathrm{a}}$ costelas, para quantificação da cobertura de gordura e área de olho do lombo, além da avaliação de cobertura de gordura na garupa. $\mathrm{O}$ delineamento experimental foi inteiramente ao acaso, sendo os resultados submetidos à análise de variância e as diferenças entre médias comparadas pelo teste $\mathrm{t}(\mathrm{P}<0,05)$ (Sampaio, 2002).

\section{RESULTADOS E DISCUSSÃO}

Os resultados para peso à desmama, peso corporal, idade e perímetro escrotal à puberdade são mostrados na Tab. 1, considerando-se como critério de puberdade a ocorrência de $50 \times 10^{6}$ espermatozoides $/ \mathrm{mL}$ por ejaculado, com no mínimo $10 \%$ de motilidade.

Tabela 1. Médias e desvios-padrão do peso à desmama (PDESM), peso corporal (PPUB), idade à puberdade (IDPUB) e perímetro escrotal (PEPUB) à puberdade em tourinhos da raça Nelore submetidos a um programa de seleção para precocidade sexual, criados em condições semi-intensivas

\begin{tabular}{ccccc}
\hline Grupo & PDESM (Kg) & IDPUB (meses) & PPUB $(\mathrm{Kg})$ & PEPUB $(\mathrm{cm})$ \\
\hline G1 (PE > 22,8cm) & $244,86 \pm 14,91$ & $13,71 \pm 0,68 \mathrm{~B}$ & $339,98 \pm 34,99 \mathrm{~B}$ & $27,06 \pm 1,35$ \\
G2 (PE 21,6 a 22,6cm) & $259,15 \pm 20,19$ & $15,89 \pm 1,53 \mathrm{~A}$ & $399,70 \pm 56,60 \mathrm{~A}$ & $25,80 \pm 3,17$ \\
G3 (PE 20,1 a 21,2cm) & $249,06 \pm 10,69$ & $16,52 \pm 0,67 \mathrm{~A}$ & $434,37 \pm 23,10 \mathrm{~A}$ & $26,37 \pm 2,32$ \\
\hline
\end{tabular}

Valores seguidos por letras maiúsculas na mesma coluna diferem entre si $(\mathrm{P}<0,05)$

Houve diferença do G1, considerado mais precoce, em relação ao G2 e ao G3 para a idade e o peso corporal à puberdade, pois ele apresentou maior $\mathrm{PE}$ à puberdade $\mathrm{e}$ menor peso à desmama em relação aos outros grupos. Em Bos taurus taurus, a puberdade foi atingida com uma PE média de 27,8cm (Lunstra et al., 1978), enquanto valores semelhantes ao do presente estudo foram descritos na raça Nelore (Vale Filho et al., 2001). Tem sido reportado que a idade à puberdade em animais da raça Nelore tem diminuído ao longo dos anos, em razão do melhoramento genético e da seleção de animais precoces, e, atualmente, características como o PE e o peso à desmama são consideradas marcadores importantes na seleção genética para precocidade sexual (Bergmann et al., 1996).

Na Fig. 1, observa-se que o G1 apresenta maior PE precocemente (12 meses), quando comparado aos outros dois grupos, o qual não aumentou de forma intensa até o fim das mensurações, mas apresentou aumento progressivo e gradual dos 12 aos 18 meses de idade. Os G2 e G3 tiveram menor PE aos 12 meses de idade em relação ao
G1, no entanto apresentaram aumento substancial entre os 12 e 14 meses de idade com o crescimento testicular estabilizando mais tardiamente nesses grupos, similar ao que ocorreu desde os 12 meses no G1.

O PE maior no G1 aos 12 meses de idade evidencia a precocidade da espermatogênese neste grupo em relação ao G2 e G3, diferença que desaparece após os 14 meses, quando todos os animais já alcançaram a puberdade e iniciaram a estabilização das características reprodutivas. Este achado assemelha-se ao descrito por França e Russell (1998), que verificaram que o crescimento testicular é rápido à medida que os animais amadurecem sexualmente, continuando o crescimento de forma mais lenta após a maturidade sexual. Além disso, verificou-se também que o perímetro escrotal no G1 foi sempre mais elevado do que nos outros grupos. Com base nesses resultados, pode-se inferir que o PE é um marcador adequado para presumir a precocidade sexual, mas não a fertilidade e a qualidade genética do animal. 


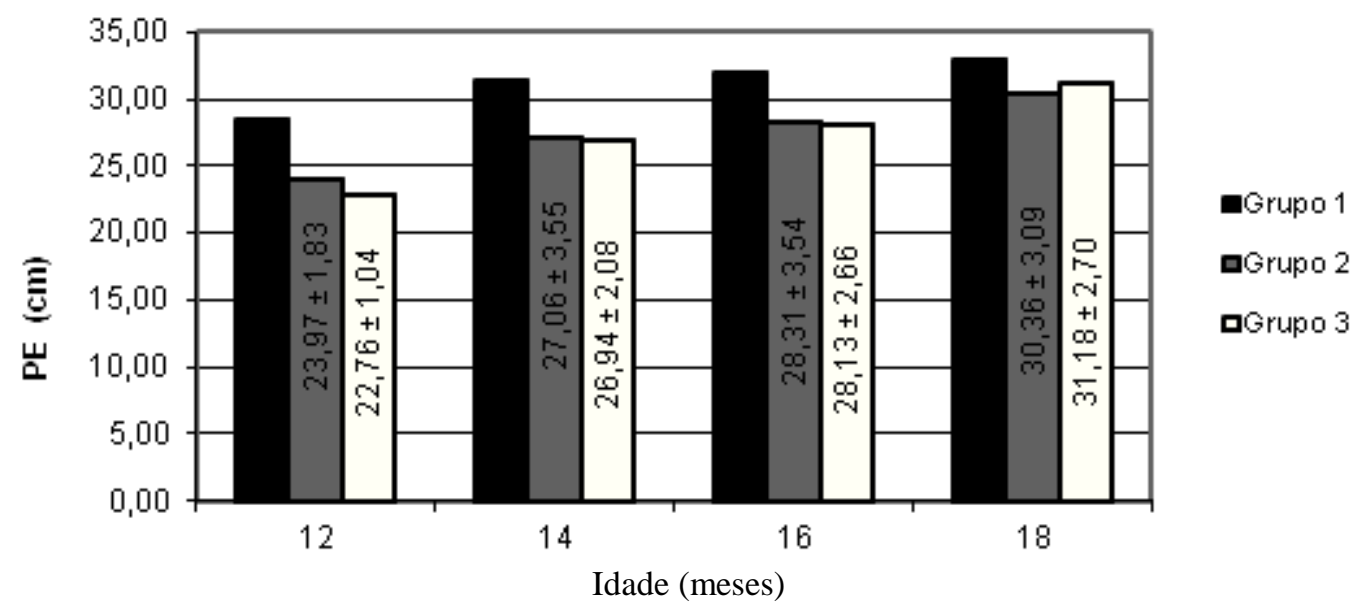

Figura 1. Perímetro escrotal (PE) de touros da raça Nelore aos 12, 14, 16 e 18 meses de idade, submetidos a um programa de seleção para precocidade sexual.

A Fig. 2 mostra aumento no peso corporal dos animais dos três grupos no período experimental. A inflexão das curvas evidencia que houve diferença na velocidade de crescimento entre os três grupos. Os animais do G1, considerados mais precoces com base na idade à puberdade $\mathrm{e}$ na avaliaçao seminal, tiveram maior ganho de peso até os 15 meses de idade em relação aos animais do G3, considerados os menos precoces. Esse resultado mostra que o maior ganho de peso ocorreu mais cedo nos animais mais precoces.

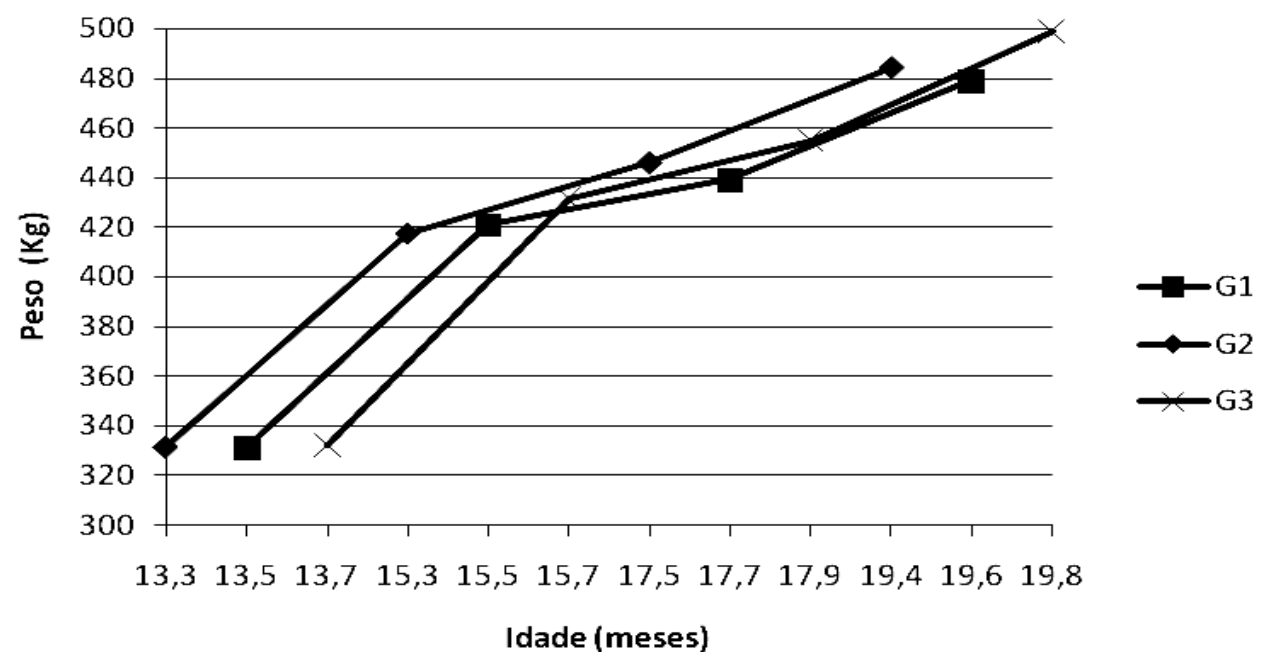

Figura 2. Peso corporal segundo a idade, em touros da raça Nelore, submetidos a um programa de seleção para precocidade sexual.

O ganho de peso diário como critério único de seleção não implica necessariamente seleção para precocidade, uma vez que se podem selecionar animais com maior peso à idade adulta. Indicativo disso são as diferenças de idade à puberdade em função do peso nas raças de corte (Barber e Almquist, 1975; Barthle e
Reiling, 1999; Barth, 2004), em que animais de raças menores, como a Angus, atingem a puberdade com peso e idade menores que aqueles de raças grandes, como Limousin e Charolês. Tais observações validam o conceito de velocidade de ganho de peso, que é a avaliação do ganho de peso do animal dentro de 
um período, compreendido geralmente do nascimento à puberdade, o que possibilita identificar animais mais precoces no rebanho (Martin et al., 1992; Pereira, 2004). É importante avaliar a razão entre o ganho de peso e o peso adulto, pois estes são diretamente proporcionais e, quanto maior o peso à idade adulta para determinada raça, maior será seu ganho de peso diário, em condições adequadas de nutrição.
Nas Tab. 2 e 3, observa-se que as medidas de AOL e EGP8 não apresentaram diferença. A homogeneidade dos animais para essas características de carcaça pode ter ocorrido pela padronização das condições experimentais, que beneficiaram os animais do experimento em relação aos outros animais do rebanho, ao terem condições mais controladas de alimentação e de ambiente, favorecendo para que essas características de carcaça fossem expressas de maneira satisfatória.

Tabela 2. Médias e desvios-padrão da área de olho de lombo (AOL), espessura de gordura (EG) e cobertura de gordura na garupa (EGP8) de touros da raça Nelore $(n=24)$ mais precoces $(\mathrm{G} 1)$ e menos precoces (G2), associados à precocidade sexual em relação à idade à puberdade

\begin{tabular}{cccc}
\hline Grupos & AOL $(\mathrm{mm})$ & EG $(\mathrm{mm})$ & EGP8 $(\mathrm{mm})$ \\
\hline G1 & $70,62 \pm 1,74 \mathrm{~A}$ & $3,20 \pm 0,20 \mathrm{~A}$ & $4,80 \pm 1,01 \mathrm{~A}$ \\
G2 & $71,64 \pm 4,64 \mathrm{~A}$ & $4,23 \pm 0,45 \mathrm{~B}$ & $4,90 \pm 0,97 \mathrm{~A}$ \\
Total & $71,09 \pm 5,26$ & $3,67 \pm 0,62$ & $4,85 \pm 0,97$ \\
\hline
\end{tabular}

Valores seguidos por letras maiúsculas na mesma coluna diferem entre si $(\mathrm{P}<0,05)$.

Tabela 3. Médias e desvios-padrão da área de olho de lombo (AOL), espessura de gordura (EG) e cobertura de gordura na garupa (EGP8) de touros da raça Nelore $(n=24)$, mais precoces $(\mathrm{G} 1)$ e menos precoces $(\mathrm{G} 2)$, associados à precocidade sexual em relação à espessura de gordura

\begin{tabular}{ccccc}
\hline Grupos & IDPUB (meses) & AOL $(\mathrm{mm})$ & EG $(\mathrm{mm})$ & EGP8 $(\mathrm{mm})$ \\
\hline G1 & $13,89 \pm 0,72 \mathrm{~A}$ & $70,49 \pm 4,31 \mathrm{~A}$ & $3,64 \pm 0,51 \mathrm{~A}$ & $4,90 \pm 0,75 \mathrm{~A}$ \\
G2 & $16,63 \pm 0,79 \mathrm{~B}$ & $71,60 \pm 6,07 \mathrm{~A}$ & $3,70 \pm 0,71 \mathrm{~A}$ & $4,80 \pm 1,15 \mathrm{~A}$ \\
Total & $15,37 \pm 1,58$ & $71,09 \pm 5,26$ & $3,67 \pm 0,62$ & $4,85 \pm 0,97$ \\
\hline
\end{tabular}

Valores seguidos por letras maiúsculas na mesma coluna diferem entre si $(\mathrm{P}<0,05)$.

Observa-se também que, ao dividir os 24 animais em dois grupos, mais precoces (G1) e menos precoces (G2), houve diferença na EG (Tab. 2) e na idade à puberdade. Como esperado, os animais do G1 foram mais precoces, porém os resultados para EG diferem do encontrado por Crews Jr. et al. (2002), que indicam que animais só começam a deposição de gordura quando já alcançaram a puberdade. No entanto, nos animais do presente experimento, a maior e mais precoce deposição de gordura ocorreu nos animais do G2, considerados mais tardios. Ainda, segundo Crews Jr. et al. (2002), a idade em que se inicia a deposição de gordura nos bovinos e sua velocidade de deposição são determinadas pelo manejo alimentar, pela maturidade, pelo sexo e pelo grupo genético do animal.

\section{CONCLUSÕES}

Com base nos resultados, conclui-se que peso corporal, perímetro escrotal e características de carcaça, como área de olho de lombo e espessura de gordura, são parâmetros adequados para seleção de touros Nelore mais precoces.

\section{REFERÊNCIAS BIBLIOGRÁFICAS}

BARBER, K.A; ALMQUIST, J.O. Growth and feed efficiency and her relationship to puberal traits of Charolais bulls. J. Anim. Sci., v.40, p.288-301,1975.

BARTH, A.D. Pubertal development of Bos taurus beef bulls. In: WORLD BUIATRICS CONGRESS, 23., 2004, Quebec, Canada. Proceedings... Quebec, Canada: [s.n.] 2004, p.11-16. 
BARTHLE, C.; REILING, A.B. Developing young bulls. In: FLORIDA BEEF CATTLE SHORT COURSE, 1999, Flórida: Quality Beef, 1999, p.59-66.

BERGMANN, J.A.G.; ZAMBORLINI, L.C.; PROCOPIO, C.S.O. et al. Estimativas de parâmetros genéticos do perímetro escrotal e do peso corporal em animais da raça Nelore. Arq. Bras. Med. Vet. Zootec., v.48, p.69-78, 1996.

BRITO, L.F.C.; SILVA, A.E.D.F.; UNANIAN, M.M. et al. Sexual development in early and late maturing Bos indicus and Bos indicus $\mathrm{x}$ Bos taurus crossbred bulls in Brazil. Theriogenology, v.62, p.1198-1217, 2004.

CREWS Jr., D.H.; SHANNON, N.H.; CREWS, R.E.; KEMP, R.A. Weaning, yearling, and preharvest ultrasound measures of fat and muscle area in steers, bulls, and heifers. J. Anim. Sci., v.80, p.2817-2824, 2002.

FERRAZ, J.B.S.; ELER, J.P. Seleção de Bos indicus para precocidade sexual. Rev. Bras. Reprod. Anim. v.31, p.167-171, 2007.

FONSECA, V.O.; CRUDELI, G.A; SILVA, E.V.C.; HERMANNY, A. Aptidão reprodutiva de touros da raça Nelore: efeito das diferentes estações do ano sobre as características seminais, circunferência escrotal e fertilidade. Arq. Bras. Med. Vet. Zootec., v.4, p.7-15, 1992.

FRANÇA, L.R.; RUSSELL, L.D. The testis of domestic animals. In: MARTINEZ, F.; REGADERA, J. (Eds.). Male reproduction: a multidisciplinary overview. Madrid,

Spain: Churchill Livingstone. 1998. p.197-219.

FRENEAU, G.E.; VALE FILHO, V.R.; MARQUES JR, A.P.; MARIA, W.S. Puberdade em touros Nelore criados em pasto no Brasil: características corporais testiculares e seminais e de índice de capacidade andrológica por pontos. Arq. Bras. Med. Vet. Zootec., v.58, p.1107-1115, 2006.

GRESSLER S.L. Estudo de fatores de ambiente e parâmetros genéticos de algumas características reprodutivas em animais da raça Nelore. 1998. 149f. Dissertação (Mestrado em Zootecnia) - Escola de Veterinária, UFMG, Belo Horizonte, MG.
LUNSTRA, D.D.; FORD, J.J.; ECHTERNKAMP S.E. Puberty in beef bulls hormone concentrations, growth, testicular development, sperm production and sexual agressiveness in bulls of different breeds. J. Anim. Sci., v.46, p.1054-1062, 1978.

MARTIN, G.B.; TJONDRONEGORO, S.; BLACKBERRY, M.A. Effects of nutrition on testicular size and the concentrations of gonadotropins, testosterone and inhibin in plasma of mature male sheep. J. Reprod. Fert., v.101, p.121-128, 1992.

PEREIRA, J.C.C. Melhoramento genético aplicado à produção animal. 4.ed. Belo Horizonte: FEP-MVZ, 2004. 252p.

QUIRINO, C.R.; BERGMANN, J.A.G.; VALE FILHO, V.R. et al. Estimativa de parâmetros genéticos para características físicas do sêmen e perímetro escrotal em touros Nelore. In: ENCONTRO DE PESQUISA DA ESCOLA DE VETERINÁRIA DA UFMG, 16., 1998, Belo Horizonte. Anais... Belo Horizonte: FEPMVZ, 1998. p.34.

SAINZ, R.D.; ARAUJO, F.R.C.; MANICARDI, F. et al. Melhoramento genético da carcaça em gado zebuíno. In: SEMINÁRIO NACIONAL DE CRIADORES E PESQUISADORES, 12., 2003, Ribeirão Preto. Anais... Ribeirão Preto: [s.n.], 2003, p.1-12.

SAMPAIO, I.B.M. Estatística aplicada à experimentação animal. 2.ed. Belo Horizonte: FEP-MVZ; 2002. p.265.

TURNER, J.W.; PELTON, L.S.; CROSS, H.R. Using live animal ultrasound measures of ribeye area and fat thickness in yearling Hereford bulls. J. Anim. Sci., v.68, p.3502, 1990.

VALE FILHO, V.R., ANDRADE, V.J.; QUIRINO, C.R. et al. Perfil andrológico de touros da raça Tabapuã (Bos taurus indicus) de um a dois anos de idade, criados extensivamente nos estados de Minas Gerais, Bahia e Espírito Santo, Brasil. In: CONGRESSO BRASILEIRO DE REPRODUÇÃO ANIMAL, 14., 2001. Belo Horizonte. Anais... Belo Horizonte: CBRA, 2001. 